\title{
HOW BÜRGI COMPUTED THE SINES OF ALL INTEGER ANGLES SIMULTANEOUSLY IN 1586
}

\author{
GRÉGOIRE NICOLLIER
}

\begin{abstract}
We present an algorithm discovered by Jost Bürgi around 1586, lost until 2013, and proven in 2015. Bürgi's method needs only sums of integers and divisions by 2 to compute simultaneously and with any desired accuracy the sines of the $n$th parts of the right angle. We explain why it works with a new proof using polygons and discrete Fourier transforms.
\end{abstract}

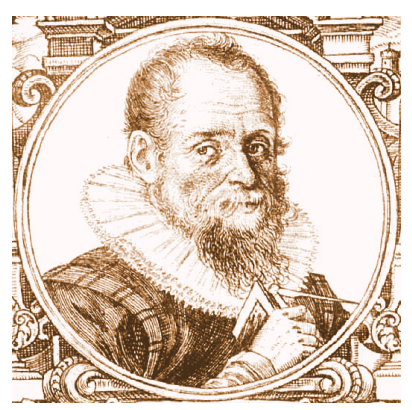

Und ${ }^{1}$ weil mir auß mangel der sprachen
die thür zu den authoribus nit alzeitt of-
fen gestanden, wie andern, hab jch etwas
mehr, als etwa die glehrte vnd belesene
meinen eigenen gedanckhen nachhengen
vnd newe wege suechen müessen.
$\quad$ Jost Bürgi, Einleitung zur Coss [13]

FIGURE 1. Jost Bürgi

(ETH Library, Zurich)

\section{A LOST MANUSCRIPT}

Menso Folkerts, now Emeritus Professor of history of sciences at the Ludwig-Maximilian University in Munich, used to visit university libraries and search for interesting old manuscripts. So did he in 1991 in Wrocław (Poland, former Breslau). After consulting the hand-written catalog of the manuscripts' collection, he ordered microfilms of several works (personal communication). Folkerts looked as late as 2013 to one of those microfilms and recognized that the autograph Fundamentum Astronomice [2], written in German by Jost Bürgi, contained the author's lost algorithm for computing sine tables (Figures 2 and 3) $[8,9]$ :

Einen rechten Winckell in also viel theile theilenn alß man will, vnnd aus demselben den Canonen Sinuum vermachenn.

Divide a right angle in as many parts as you want and construct herefrom the sine table.

Date: October 31, 2017. The final publication in Math. Semesterber. 65 (2018) 15-34 is available at http://link.springer.com, doi:10.1007/s00591-017-0209-0.

Key words and phrases. Jost Bürgi · Sine table · Discrete Fourier transformation · Finite difference · Planar polygon · Napoleon's theorem · Petr-Douglas-Neumann theorem.

We are very grateful to Jörg Waldvogel for his generous and fruitful introduction to the subject.

${ }^{1}$ And as, because of my lack of languages, the door to the authors wasn't always open to me as it was to others, I had to follow my own thoughts and find new ways a little more than, for example, the scholars and well-read. Jost Bürgi, Introduction to Coss [13]. 


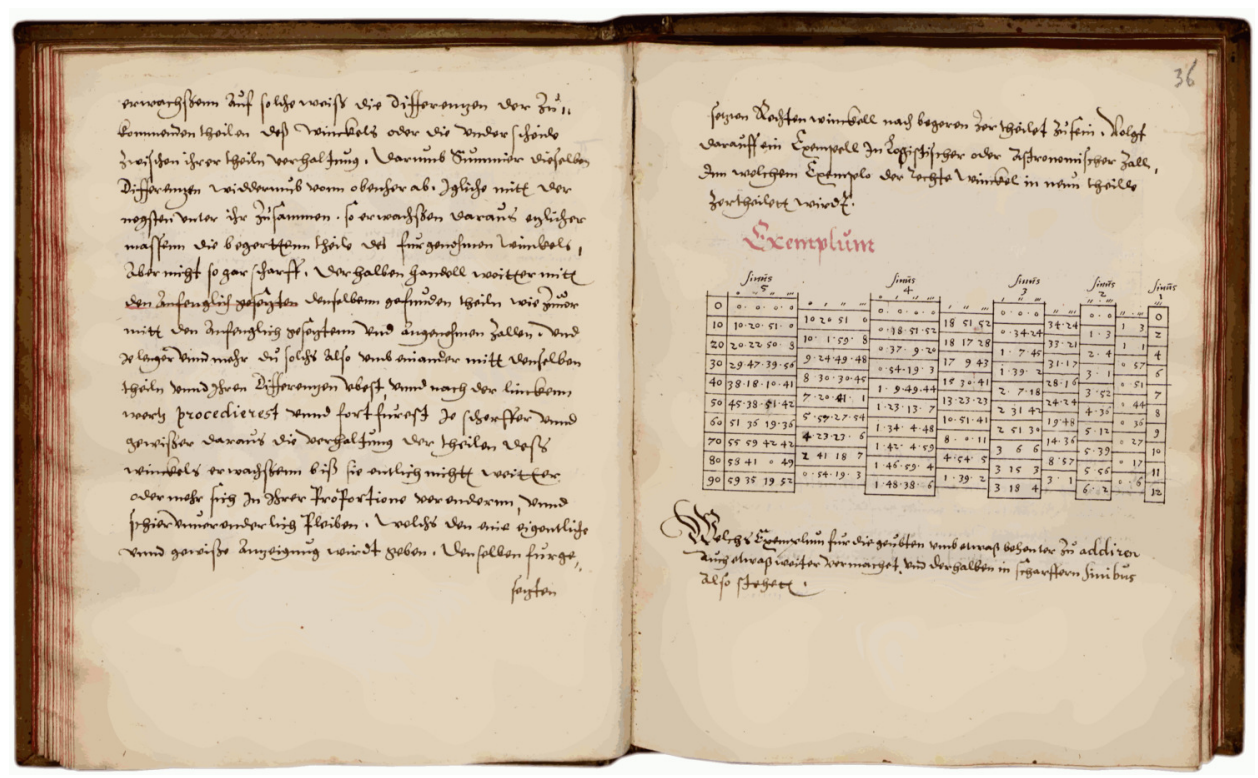

FIGURE 2. Fundamentum Astronomice (University Library, Wrocław [2])

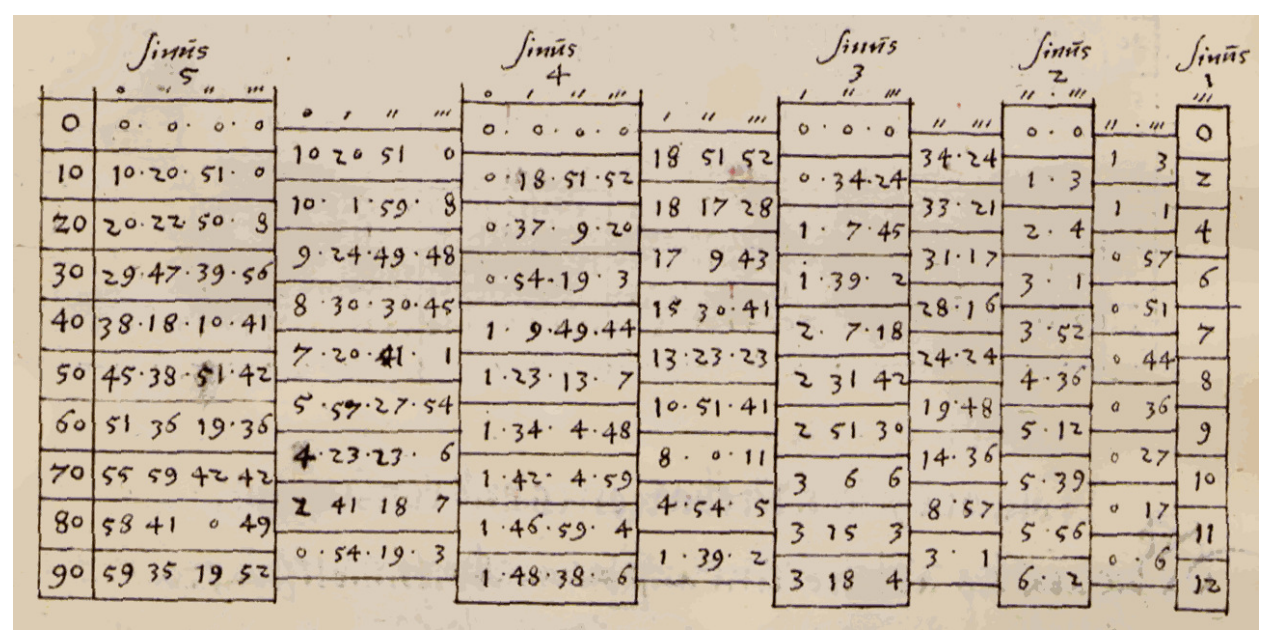

FIGURE 3. Artificium (University Library, Wrocław [2])

Bürgi's example of his skillful method, the Artificium, explains the calculation for the multiples of $10^{\circ}$, the ninth parts of the right angle. If we leave away zeros and column shifts in Figure 3, write sexagesimal numbers in base ten (for example $1^{\prime \prime} 3^{\prime \prime \prime}=1 \times 60+3=63$ at the top of the next to rightmost column), and rotate the table by a quarter-turn, we obtain 
the following first three lines from the three rightmost columns Sinus 1 to Sinus 2:

$\begin{array}{lrrrrrrrrr}\text { (a) } & 2 & 4 & 6 & 7 & 8 & 9 & 10 & 11 & 12 \\ \text { (b) } & 63 & 61 & 57 & 51 & 44 & 36 & 27 & 17 & 6 \\ \text { (c) } & 63 & 124 & 181 & 232 & 276 & 312 & 339 & 356 & 362\end{array}$

The Bürgi step. (a) Start with a finite sequence, (b) halve the last entry and take the cumulative sums from the right, and (c) from the left.

The example of Figure 3 consists of four iterated Bürgi steps and is produced by three more. The initial and last lines of each step divided by their rightmost entry, which is the circle radius, provide sine values for every $10^{\circ}$ that converge to the correct values: the errors are here smaller than $2.5 \times 10^{-7}$ after four steps and smaller than $1.9 \times 10^{-11}$ three steps later. Only sums of integers and halving are involved before the final divisions and all sine values are determined simultaneously. The Artificium and the whole autograph were decrypted and edited by Dieter Launert [12,9]. The first proof of convergence was given by Andreas Thom using the Perron-Frobenius theorem [9]. Another proof with matrices by Jörg Waldvogel is more elementary and determines the rate of convergence [30].

In this paper we present a new proof of convergence that encompasses a whole family of related methods, in particular the following two.

The Darboux-Nicollier step. (a) Start with a finite sequence, (b) add to each entry the left neighbor, if any, and (c) add to each entry the right neighbor, if any, and double the last entry:

$\begin{array}{lrrrrrrrrr}\text { (a) } & 2 & 4 & 6 & 7 & 8 & 9 & 10 & 11 & 12 \\ \text { (b) } & 2 & 6 & 10 & 13 & 15 & 17 & 19 & 21 & 23 \\ \text { (c) } & 8 & 16 & 23 & 28 & 32 & 36 & 40 & 44 & 46\end{array}$

To get directly from (a) to (c), add the direct neighbors to twice each entry by considering the left neighbor of the last entry also as its right neighbor.

The inverse Bürgi step. Replace in the Darboux-Nicollier step 'add to' with 'subtract from' (example in Table 1).

Here is the main result.

Theorem 1. If one starts with any complex sequence $\left(s_{j}\right)_{1 \leq j \leq n}$ of length $n \geq 1$ such that

$$
\sum_{j=1}^{n-1} s_{j} \sin \frac{j \pi}{2 n}+\frac{1}{2} s_{n} \neq 0
$$

(for example if the $s_{j}$ are real, nonnegative, and not all zero), the sequence obtained after $m$ Bürgi or Darboux-Nicollier steps and divided by its last entry converges for $m \rightarrow \infty$ to the table $\left(\sin \frac{j \pi}{2 n}\right)_{1 \leq j \leq n}$ containing the sines of the right angle's nth parts. The middle line of the mth step divided by its first entry tends then to $\sec \frac{\pi}{4 n} \cdot\left(\cos \frac{(2 j-1) \pi}{4 n}\right)_{1 \leq j \leq n}$ in the Bürgi case and to $\csc \frac{\pi}{4 n} \cdot\left(\sin \frac{(2 j-1) \pi}{4 n}\right)_{1 \leq j \leq n}$ in the Darboux-Nicollier case, which are proportional to sine tables of the right angle's intermediate $(2 n)$ th parts.

If the initial sequence fulfills

$$
\sum_{j=1}^{n-1}(-1)^{j} s_{j} \sin \frac{j \pi}{2 n}+\frac{(-1)^{n}}{2} s_{n} \neq 0,
$$

the last and middle lines of the mth inverse Bürgi step divided by their last entry converge to $\left((-1)^{n+j} \sin \frac{j \pi}{2 n}\right)_{1 \leq j \leq n}$ and $\sec \frac{\pi}{4 n} \cdot\left((-1)^{n+j} \sin \frac{(2 j-1) \pi}{4 n}\right)_{1 \leq j \leq n}$. 
As mentioned above, the convergence of the original Bürgi algorithm was already proven in $[9,30]$. The other assertions of Theorem 1 are new. Notice however that Launert remarks without proof [12, pp. 55 and 57] that the middle line of the Bürgi step divided by its first entry can be used to approximate the cosine values $\cos \frac{(2 j-1) \pi}{4 n}$, but the necessary factor $\sec \frac{\pi}{4 n}$ is missing there! Our proof of Theorem 1, in Section 5, is matrix-free and relies on polygons and discrete Fourier transformation. We will in fact consider this theorem as a special case of a more general result valid for any suitably normalized operator that is diagonal in the Fourier basis, commutes with complex conjugation, and has for example two simple dominating eigenvalues (Equation (3) and Theorem 2). We will further improve the rate of convergence of the iterated Bürgi steps by choosing the initial sequence appropriately (Section 6).

\section{JOST BÜRGI}

Jost Bürgi (1552-1632) was born in Lichtensteig (Toggenburg) near St. Gallen in Switzerland. (All historical informations are borrowed from [13, 4, 8, 12, 9, 28] and Kepler's collected works [11].) Bürgi's life took place in religiously troubled times: Protestant Reformation and Roman Inquisition-both impacting on astronomy by rejecting the heliocentric model - and Thirty Years' War (1618-1648). Europe was also suffering at that time from epidemies and from increasingly cold weather and poor crops in connection with the Little Ice Age. Bürgi became a clockmaker, but we do not know where he acquired his skills: very little is ascertained about his life prior to 1579 when he began at the court of Landgrave William IV (1532-1592) in Cassel, with a high salary, as clock and instrument maker in the observatory. He stayed there until 1603 before moving to the imperial court in Prague. Turning definitely back to Cassel in 1631, he died a few months later, survived for two weeks by his second wife and without own children.

The young Bürgi built in Cassel his first celestial globe and developed precision instruments (sextants, compasses, a clock working three months without winding). He invented in 1584 the first clock with second precision and a hand for seconds as well as an acoustic signal, which allowed Tycho Brahe (1546-1601), Bürgi himself, other astronomers, and later Johannes Kepler (1571-1630) to improve their observations. Tycho Brahe, who had close contacts with Cassel, used to avoid naming Bürgi in his correspondence and referred to him disdainfully as the automatopæus (instrument maker).

Accurate sine tables were vital for astronomy. Moreover, before the introduction of logarithms, they enabled computing a product as a sum with the so-called prosthaphaeresis,

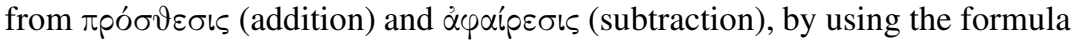

$$
\cos \alpha \cos \beta=\frac{\cos (\alpha+\beta)+\cos (\alpha-\beta)}{2}
$$

or an equivalent one. A sine table for every minute would give without interpolation

$$
5.163 \times 6.281 \approx 10^{2} \times \cos 58^{\circ} 55^{\prime} \times \cos 51^{\circ} 5^{\prime}=10^{2} \times \frac{\cos 110^{\circ}+\cos 7^{\circ} 50^{\prime}}{2} \approx 32.43 .
$$

A table of cosecants was needed for divisions. Bürgi, an autodidact mathematician, rediscovered with others the prosthaphaeresis well-known to Indian and Arabic astronomers of the Middle Ages. As Bürgi spoke neither Latin nor Greek (see the introductory citation), he had a great handicap to publishing his mathematical discoveries.

Fundamentum Astronomia [2], Bürgi's first work, is a treatise on sexagesimal arithmetic and plane and spherical trigonometry of about two hundred pages $($ c. $19.5 \times 15 \mathrm{~cm})$ 


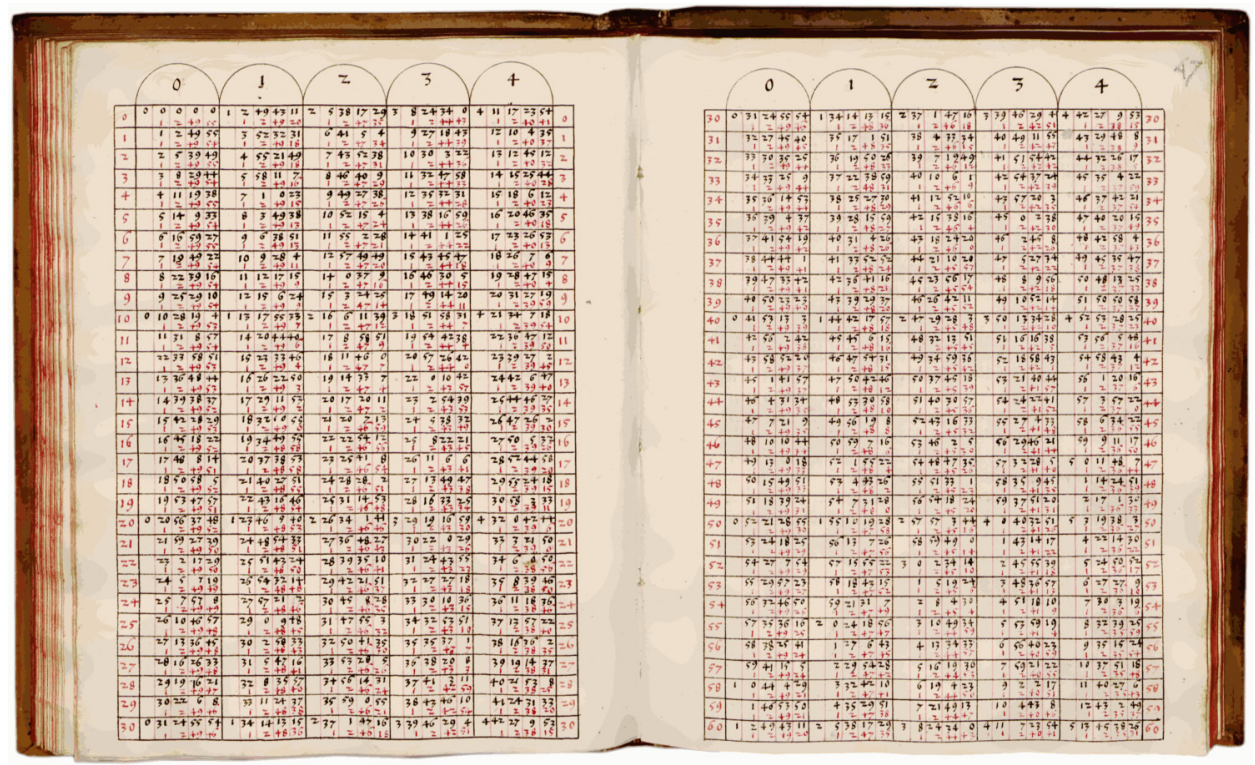

FIGURE 4. Canon sinuum: double page from $0^{\circ}$ to $5^{\circ}$ (University Library, Wrocław [2])

written from c. 1586 onwards that contains a sine table. This Canon sinuum in sexagesimal numbers for every minute of the right angle fills thirty-six pages (Figure 4). Although novel procedures are described in the manuscript, it is not always clear to which extent they were really used for the Canon's computation and how much Bürgi worked with Ptolemy's classical method he also describes [12, 9, 24] ([24] contains references to other reports by Denis Roegel). Probably, the sines of the degrees from $0^{\circ}$ to $90^{\circ}$ provided by the Artificium served as pivots for the refinement. With the help of usual trigonometric identities and an accurate value of $\sin 1^{\prime}$ obtained by correcting upwards the sixtieth part of $\sin 1^{\circ}$, Bürgi determined values of $\sin \left(d^{\circ}+m^{\prime}\right)$ for the first three minutes $m^{\prime}$ of every degree $d^{\circ}$. The sine function was then extended from the values for $m=0,1,2,3$ to the whole interval $\left[d^{\circ},(d+1)^{\circ}\right]$ by a cubic polynomial extrapolation: the third difference computable from the known initial values was supposed constant for the rest of the interval and used to fill in backwards the missing differences, from the second to the zeroth order. The first differences were included in the sine table in order to facilitate linear interpolation. Bürgi noticed that it suffices to compute the sine table from $0^{\circ}$ to $30^{\circ}$ to get $\left[60^{\circ}, 90^{\circ}\right]$ with the Pythagorean trigonometric identity and then $\left[30^{\circ}, 60^{\circ}\right]$ from $\sin \alpha=\sin \left(60^{\circ}+\alpha\right)-\sin \left(60^{\circ}-\alpha\right)$, but it is incertain whether he did so.

The existence of Bürgi's sine algorithm was known from a cryptic mention by Nicolaus Reimers Bær (Ursus, 1551-1600) in his Fundamentum astronomicum (1588): he had promised to Bürgi, the friend and teacher he met when living in Cassel during 1586-87, that he would keep the Artificium secret until publication, which never occurred! The method was nevertheless probably in possession of Henry Briggs, whose name appears together with a computed example (hand-written c. 1620 by an unknown reader) in an exemplar of Ursus' book now at the University of Leiden [12,9]. The sine table for every $2^{\prime \prime}$, presumably completed by Bürgi in 1598, is lost. 


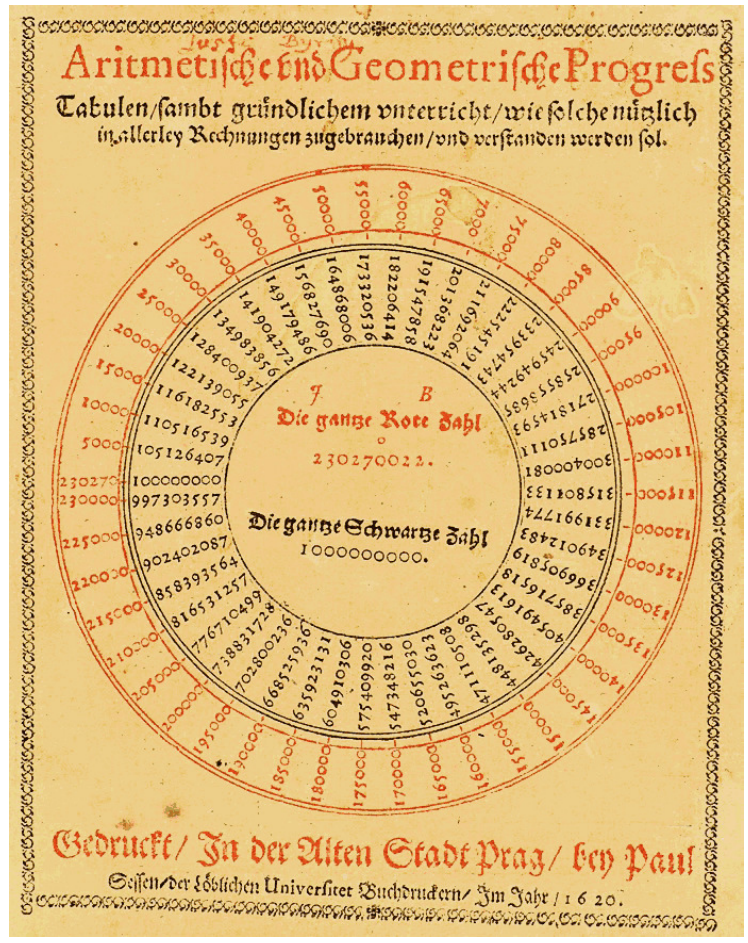

FIgURE 5. Front page of Progreß Tabulen (University Library, Graz)

In 1592, Bürgi delivered a planetary globe of his own to the orderer, Holy Roman Emperor Rudolf II von Habsburg (1552-1612), who lived in Prague, and gave him as a present the dedicated and unique exemplar of Fundamentum Astronomiae.

Maybe already in 1588 and certainly before 1610, Bürgi computed the very first table of logarithms (in fact a table of exponentials) [4]: he tabulated the natural powers of 1.0001, multiplied by $10^{8}$ and rounded to integers, until $1.0001^{23027} \approx 9.99999779$ and computed correctly by linear extrapolation

$$
1.0001^{23027.0022}<10<1.0001^{23027.0023} .
$$

But he published this table as late as 1620 in a very limited edition (Figure 5) after John Napier's table (1614, Napierian logarithms given by the power of $1-10^{-7}$ equal to the sine of the angle) and Henry Briggs' table (1617 and 1624, common logarithms). Only two complete original exemplars of Bürgi's Aritmetische und Geometrische Progreß Tabulen are still extant: the printed logarithm table with separate hand-written instructions for use. The front page of Figure 5 summarizes the results in a way very similar to a circular slide rule(!) with exponents printed in red and contains one misprint: it should be '5000 105126847' since $10^{8} \times 1.0001^{5000 / 10} \approx 105126847$. (The entry ' $23027010^{8}$ ' is correct if one considers the fact that 230270 and 0 occupy the same place.)

Intensive astronomic measurements, orbit computations, constructions of globes, clocks, and instruments continued under William's successor, his son Landgrave Moritz I. About 1600, Bürgi was preparing his Arithmetica Bürgii, also called the Coss, as an introduction to his $2^{\prime \prime}$ sine table. Kepler received—probably from Ursus, Rudolf's imperial 
mathematician - a draft of this project, formulated it to a final text [13], promising to keep it secret, and gave it back to Bürgi in 1603, who never published it. Coss, from the Italian word cosa for 'thing', was the name of the unknown in an algebraic problem and denoted by extension the theory of equations in early symbolic algebra.

Having been banished from Graz for refusing to convert to Catholicism, Kepler was since 1601 imperial mathematician (and as such, astrological advisor) at the court of Rudolf II (a Catholic!), replacing his dead predecessor and concurrent Tycho Brahe. He was joined in 1603 by Bürgi, new imperial clockmaker one year later and third most-paid employee of Rudolf. In De stella tertii honoris in Cygno from 1606 [11, Vol. I, p. 308], Kepler praised his colleague in the highest tones.

Alter quem ego novi, est Jvstvs BYRGivs, S. C. Majest. Automatopœus; qui licet expers linguarum, rerum tamen Mathematicarum scientiâ et speculatione, multos earum Professores facilè superat. Praxin verò sic peculiariter sibi possidet; ut habitura sit posterior æatas, quem in hoc genere Coryphæum celebret, non minorem quàm DVRERVM in pictoria, cujus crescit occulto, velut arbor, ævo fama.

I also know Justus Byrgius, the imperial instrument maker. Although deprived of languages, he easily surpasses many professors of mathematics in his knowledge and intense study of this matter. He possesses a practical skill so peculiar, in fact, that a future generation will celebrate him as a leader in this field as much as they celebrate Dürer in painting, whose fame, like a tree, grows in a hidden way through the ages [from Horace, Carmina I.12, l. 45].

By contrast, Kepler was less friendly in 1627 in the introduction of Tabula Rudolphiance [11, Vol. X, p. 48].

[...] qui etiam apices logistici JVsto BYRGiO multis annis ante editionem Neperianam viam præiverunt ad hos ipsissimos Logarithmos. Etsi homo cunctator et secretorum suorum custos fœtum in partu destituit, non ad usus publicos educavit.

The logistic apices [that is, the indices ${ }^{\circ},{ }^{\prime},{ }^{\prime}, \ldots$ used in sexagesimal numbers] also showed Jost Bürgi, many years before Napier's edition, the way to even the same Logarithms. But this lingerer and secretmonger abandoned his child at birth and did not educate him for the public use.

Johannes Kepler being short-sighted with one eye's cornea scarred as an aftereffect of smallpox, Bürgi was his devoted observer, measurer, and provider of very accurate instruments-for example during the discovery of Kepler's Supernova in 1604-until Kepler leaved Prague for Linz in 1612. Astronomia Nova de motibus stellae Martis with Kepler's first two laws appeared in 1609.

The unique known portrait of Bürgi shows him in 1619, on the day of his 67th anniversary (Figure 1).

\section{A hypothetical GENESis of the ARTificium}

We consider doubly infinite complex sequences $\left(z_{j}\right)_{j \in \mathbf{Z}}$ as discrete-time signals and operators on these signals as filters that transform an input signal into an output. To deal with the Bürgi filter and its inverse in our proof of the Artificium and its generalizations we need the right and left difference filters

$$
\partial_{r}:\left(z_{j}\right)_{j} \mapsto\left(z_{j}-z_{j+1}\right)_{j} \quad \text { and } \quad \partial_{\ell}:\left(z_{j}\right)_{j} \mapsto\left(z_{j}-z_{j-1}\right)_{j}
$$

and the second difference filter

$$
\partial_{2}=\partial_{r} \circ \partial_{\ell}=\partial_{\ell} \circ \partial_{r}:\left(z_{j}\right)_{j} \mapsto\left(2 z_{j}-z_{j-1}-z_{j+1}\right)_{j} .
$$

(Note that the classical second central difference operator is $-\partial_{2}$.)

The Darboux filter relies on the right and left sum filters

$$
\sigma_{r}\left(z_{j}\right)_{j}=\left(z_{j}+z_{j+1}\right)_{j} \text { and } \sigma_{\ell}\left(z_{j}\right)_{j}=\left(z_{j}+z_{j-1}\right)_{j}
$$




\begin{tabular}{rllll|llllllllll|lll}
\hline Position $j=$ & $\ldots$ & -2 & -1 & 0 & 1 & 2 & 3 & 4 & 5 & 6 & 7 & 8 & 9 & 10 & 11 & $\ldots$ \\
$S=\left(s_{j}\right)_{j}=$ & $\ldots$ & -4 & -2 & 0 & 2 & 4 & 6 & 7 & 8 & 9 & 10 & 11 & 12 & 11 & 10 & $\ldots$ \\
& $\downarrow$ & $\partial_{\ell}$ & & & & $\mid$ & & & & & & & & & & & $\int_{\ell} \uparrow$ \\
& $\ldots$ & $\ldots$ & 2 & 2 & 2 & 2 & 2 & 1 & 1 & 1 & 1 & 1 & 1 & -1 & -1 & $\ldots$ \\
& $\downarrow$ & $\partial_{r}$ & & & & & & & & & & & & $\times 2$ & & \\
$\partial_{2} S \quad$ & $\ldots$ & $\ldots$ & 0 & 0 & 0 & 0 & 1 & 0 & 0 & 0 & 0 & 0 & 2 & 0 & $\ldots$ & $\ldots$ \\
\hline
\end{tabular}

TABLE 1. Second difference filter applied to a pseudo-sine of period 36

and the second sum filter

$$
\sigma_{2}=\sigma_{r} \circ \sigma_{\ell}=\sigma_{\ell} \circ \sigma_{r}:\left(z_{j}\right)_{j} \mapsto\left(2 z_{j}+z_{j-1}+z_{j+1}\right)_{j}
$$

(The mean filters $\sigma_{r} / 2, \sigma_{\ell} / 2$ would also do the job, see Figure 8!)

It is geometrically immediate that the signals $\left(e^{i j 2 \omega}\right)_{j}$ are eigenfunctions of these filters with corresponding eigenvalues

$$
\begin{array}{rr}
2 \sin \omega \cdot e^{ \pm i\left(\omega-\frac{\pi}{2}\right)} \text { for } \partial_{r} \text { and } \partial_{\ell}, & 4 \sin ^{2} \omega \text { for } \partial_{2}, \\
2 \cos \omega \cdot e^{ \pm i \omega} \text { for } \sigma_{r} \text { and } \sigma_{\ell}, & 4 \cos ^{2} \omega \text { for } \sigma_{2} .
\end{array}
$$

As the eigenvalues for $\partial_{2}$ and $\sigma_{2}$ are real and even, they are also eigenvalues belonging to $\cos j 2 \omega$ and $\sin j 2 \omega$, the real and imaginary parts of $e^{i j 2 \omega}$. The corresponding trigonometric identities were well-known to Bürgi, for example

$$
2 \sin j 2 \omega-\sin (j-1) 2 \omega-\sin (j+1) 2 \omega=4 \sin ^{2} \omega \cdot \sin j 2 \omega .
$$

Taking $2 \omega=\frac{\pi}{18}$, Bürgi thus knew that the 36 -periodic sine sequence $\left(\sin \frac{j \pi}{18}\right)_{j}$ is proportional to its image under $\partial_{2}$. And he probably wondered: "What happens if I start from a sequence only approximatively proportional to this sine sequence? Let us try with the pseudo-sine $S=\left(s_{j}\right)_{j}$ given by $\left(s_{j}\right)_{1 \leq j \leq 9}=(2,4,6,7,8,9,10,11,12)$ and for the rest odd with respect to index $j=0$ and even with respect to $j=9$."

Definition 1 (Pseudo-sines and pseudo-cosines). A pseudo-sine of period $4 n$ is a sequence odd with respect to index $j=0$ and even with respect to $j=n$, whereas a pseudo-cosine of period $4 n$ is even with respect to 'index' $j=\frac{1}{2}$ and odd with respect to 'index' $j=n+\frac{1}{2}$. The core of a pseudo-sine or pseudo-cosine is the portion indexed from 1 to $n$.

The decomposition $\partial_{2}=\partial_{r} \circ \partial_{\ell}$ applied to $S$ leads to Table 1 . Notice that $\partial_{\ell}$ transforms a pseudo-sine into a pseudo-cosine and $\partial_{r}$ a pseudo-cosine into a pseudo-sine. Let us return to Bürgi's hypothetical thoughts: "The pseudo-sine $\partial_{2} S$ is far less proportional to the sine sequence than $S$ ! But proportionality is of course dramatically improved by the inverse procedure from $\partial_{2} S$ to $S$. And what about the iterated inverse procedure applied to $S$ ? Proportionality becomes better with every iteration step (Figure 3 ) and this is for sure true in general!" After $m$ steps, the Bürgi filter outputs thus a pseudo-sine given by an iterated double integral $\left(\int_{\ell} \circ \int_{r}\right)^{\circ m} S$ of the initial pseudo-sine and these successive outputs become purer and purer, so that they converge to a sine wave when normalized. For a pseudo-sine input, the description of the Bürgi filter can be restricted to the cores of the involved pseudo-sines and pseudo-cosine, due to their symmetries: this is the Bürgi step! (We will extend the Bürgi filter to all polygons.) 

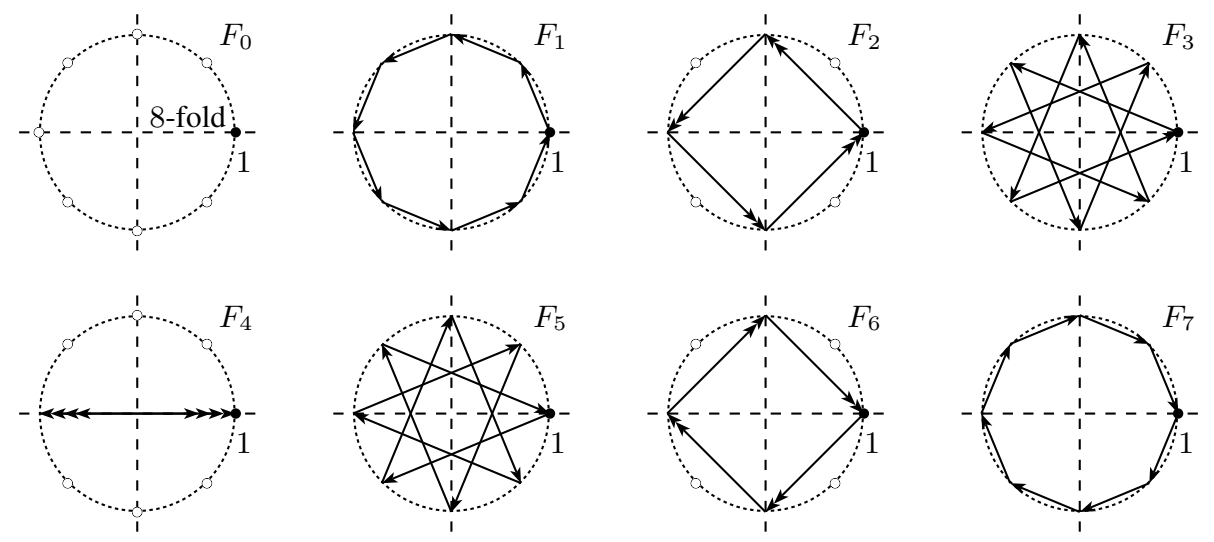

FIGURE 6. Fourier basis of $\mathbf{C}^{8}$

We in fact only need periodic signals. For an integer $N \geq 2$, an $N$-periodic signal $\left(z_{j}\right)_{j \in \mathbf{Z}}$ can be advantageously considered as an $N$-gon $P$ in the complex plane given by the finite sequence

$$
P=\left(z_{j}\right)_{0 \leq j \leq N-1}
$$

of its vertices $z_{j}$ taken in order, representing the closed polygonal line

$$
z_{0} \rightarrow z_{1} \rightarrow \cdots \rightarrow z_{N-1} \rightarrow z_{0}
$$

starting at $z_{0}$. The vertices are indexed modulo $N$. The polygon is central if the sum of its vertices is zero.

\section{THE FOURIER DECOMPOSITION}

The Fourier basis of $\mathbf{C}^{N}$ (Figure 6) is constituted by the standard regular $N$-gons

$$
F_{k}=\left(e^{i j k 2 \pi / N}\right)_{0 \leq j \leq N-1}, \quad k=0,1, \ldots, N-1 .
$$

After the starting vertex 1 , each vertex of $F_{k}$ is the $k$ th next $N$ th root of unity. $F_{0}=$ $(1,1, \ldots, 1)$ is a trivial polygon and the other basis polygons are central with $\overline{F_{k}}=F_{N-k}$ : $F_{N-k}$ is $F_{k}$ the other way around! When $N$ is even, one has $F_{N / 2}=(1,-1, \ldots, 1,-1)$. The Fourier basis is a basis as it is orthonormal with respect to the inner product of $\mathbf{C}^{N}$ given by

$$
\langle P, Q\rangle=\left\langle\left(z_{j}\right),\left(w_{j}\right)\right\rangle=\frac{1}{N} \sum_{j=0}^{N-1} z_{j} \overline{w_{j}}
$$

The discrete Fourier transform or spectrum of $P$ is the polygon $\widehat{P}=\left(\hat{z}_{k}\right)_{0 \leq k \leq N-1}$ given by the spectral decomposition of $P$ in the Fourier basis:

$$
P=\sum_{k=0}^{N-1} \hat{z}_{k} F_{k} \quad \text { with } \quad \hat{z}_{k}=\left\langle P, F_{k}\right\rangle, \quad 0 \leq k \leq N-1,
$$

where each $\hat{z}_{k} F_{k}$ is the image of $F_{k}$ under a spiral similarity about the origin (Figure 7). The trivial polygon $\hat{z}_{0} F_{0}$ corresponds to the (vertex) centroid $\hat{z}_{0}$ of $P$ : it vanishes if and only if $P$ is central.

In other words, the discrete $N$-periodic oscillation $P$ is the superposition of its pairwise orthogonal affinely $k$-regular components $A_{k}$ given by the offset $A_{0}=\hat{z}_{0} F_{0}$ and the central 


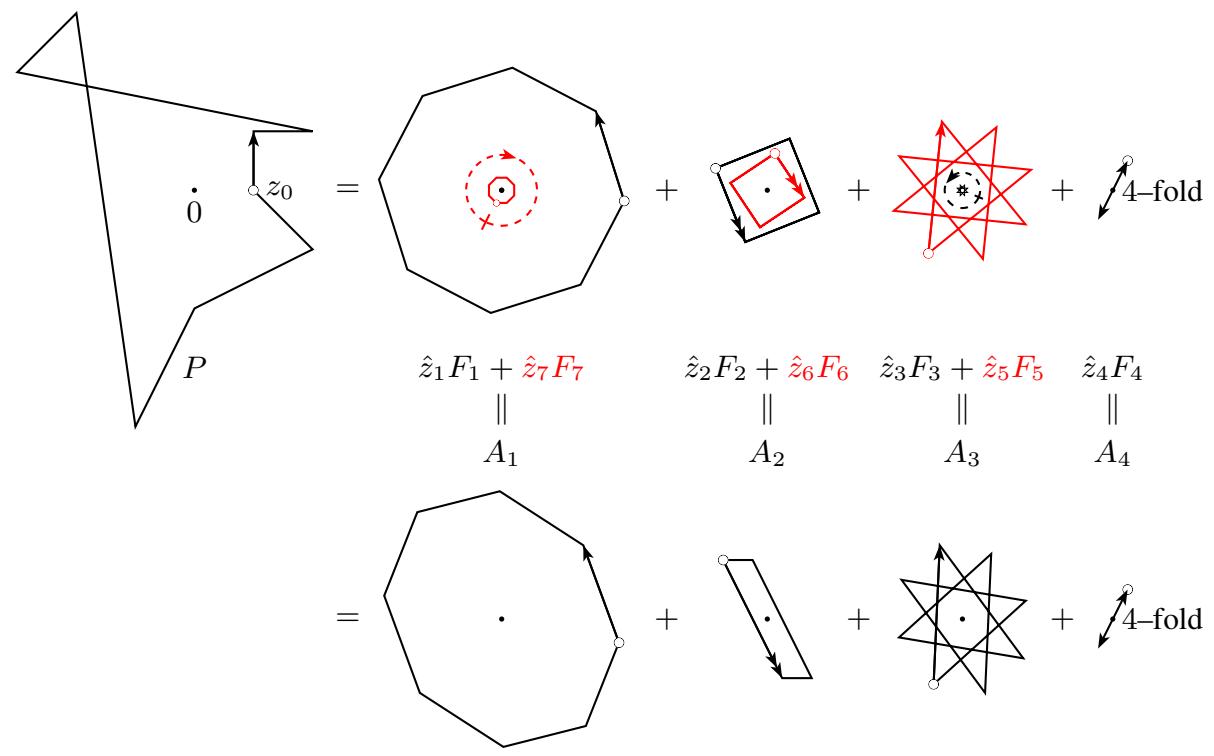

FIGURE 7. Spectral decomposition of a central octagon

harmonics $A_{k}=\hat{z}_{k} F_{k}+\hat{z}_{N-k} F_{N-k}, 1 \leq k<N / 2$, and $A_{N / 2}=\hat{z}_{N / 2} F_{N / 2}$ when $N$ is even, the first harmonic $A_{1}$ being the fundamental and the higher harmonics the overtones (Figure 7). Each harmonic has a circumellipse (Remark 1). When $P$ is real, one has

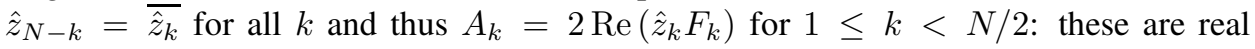
harmonic oscillations sampled at integer times.

The use of discrete Fourier transforms in the geometry of polygons goes back to Darboux in 1878, who studied the iterated polygons constructed on the side midpoints [5]. This very powerful tool often leads to one-line proofs $[1,7,10,17,18,19,20,21,22,25$, 26, 27, 29] (more references can be found in [17]).

\section{OUR SPECTRAL PROOF OF THE ARTIFICIUM}

The above difference and sum filters are linear and preserve sequence periodicity. By (1) for $\omega=\frac{k \pi}{N}$, they are diagonal in the Fourier basis. The corresponding eigenvalues $H_{k}=$ $g_{k} e^{i \eta_{k}}$ are the frequency response(s) of the filter (its spectrum), their moduli $g_{k}$ being the filter's $\operatorname{gain}(s)$ :

$$
\begin{aligned}
2 \sin \frac{k \pi}{N} \cdot e^{ \pm i\left(\frac{k}{N}-\frac{1}{2}\right) \pi} \text { for } \partial_{r} \text { and } \partial_{\ell}, & 4 \sin ^{2} \frac{k \pi}{N} \text { for } \partial_{2}, \\
2 \cos \frac{k \pi}{N} \cdot e^{ \pm i \frac{k \pi}{N}} \text { for } \sigma_{r} \text { and } \sigma_{\ell}, & 4 \cos ^{2} \frac{k \pi}{N} \text { for } \sigma_{2}, \quad 0 \leq k \leq N-1 .
\end{aligned}
$$

Each pair $\partial_{r}, \partial_{\ell}$ and $\sigma_{r}, \sigma_{\ell}$ consists of adjoint filters, that is, with complex conjugate frequency responses. Moreover the four filters clearly commute with complex conjugation: this means $H_{N-k}=\overline{H_{k}}$ for all $k$.

The kernel of $\partial_{r}$ and $\partial_{\ell}$ is the linear span of $F_{0}$. Let $\int_{r}$ and $\int_{\ell}$ be the adjoint filters whose frequency responses are given by 0 for $F_{0}$ and otherwise by the inverses of the frequency responses (2) of $\partial_{r}$ and $\partial_{\ell}$, respectively: $\partial_{r}$ and $\partial_{\ell}$ are invertible on the subspace of central $N$-gons with inverses $\int_{r}$ and $\int_{\ell}$. If $S$ and $C$ are a pseudo-sine and a pseudo-cosine, hence 
central, the first and second transformations of the Bürgi step are reversed by $\partial_{r}$ and $\partial_{\ell}$, respectively: the explicit transformations of $S$ and $C$ in the Bürgi step are thus indeed these spectrally defined $\int_{r} S$ and $\int_{\ell} C$.

We want to unify the treatment of the Bürgi, Darboux, and inverse Bürgi filters. After suitable normalization, they all can be lumped together into the following class of admissible filters.

Definition 2 (Admissible filter). A filter $\varphi$ defined on $N$-gons for some $N \geq 2$ and diagonal in the Fourier basis $\left\{F_{k}, 0 \leq k \leq N-1\right\}$ is called admissible if it commutes with complex conjugation and the maximal gain 1 is attained for $k \leq\lfloor N / 2\rfloor$ only once, say by $F_{k_{1}}$. We denote the adjoint filter by $\varphi^{*}$ and set $\tau_{1}=\varphi, \tau_{2}=\varphi^{*} \circ \varphi$, and $\tau_{2 m}=\left(\varphi^{*} \circ \varphi\right)^{\circ m}=\tau_{2}^{\circ m}, \tau_{2 m+1}=\varphi \circ\left(\varphi^{*} \circ \varphi\right)^{\circ m}=\tau_{1} \circ \tau_{2 m}$ for all natural $\mathrm{m}$.

We consider now an $N$-gon $P=\left(z_{j}\right)_{j}=\sum_{k=0}^{\lfloor N / 2\rfloor} A_{k}$ and an admissible filter $\varphi$ with frequency response $H_{k}=g_{k} e^{i \eta_{k}}, H_{N-k}=\overline{H_{k}}$. The fact that the filter commutes with complex conjugation implies that each harmonic and its image under $\varphi$ share a common circumellipse, as is shown in the following Remark 1.

Remark 1 (Circumellipses). As $A_{k}=\hat{z}_{k} F_{k}+\hat{z}_{N-k} \overline{F_{k}}$ and $\varphi A_{k}=\hat{z}_{k} H_{k} F_{k}+\hat{z}_{N-k} \overline{H_{k} F_{k}}$ for $1 \leq k<N / 2$, the real-linear complex transformation $z \mapsto \hat{z}_{k} z+\hat{z}_{N-k} \bar{z}$ maps $F_{k}$ to $A_{k}, H_{k} F_{k}$ to $\varphi A_{k}$, and $\overline{H_{k}} F_{k}$ to $\varphi^{*} A_{k}$ : since the unit circle is mapped to an ellipse, the corresponding harmonics $\varphi A_{k}$ of $\varphi P$ and $\varphi^{*} A_{k}$ of $\varphi^{*} P$ have the same circumellipse centered at 0 , which has the same axes as the circumellipse of $A_{k}$ (and this is also trivially true for $A_{N / 2}$ and $\varphi A_{N / 2}$ when $N$ is even). As $H_{k_{1}}=e^{i \eta_{k_{1}}}, A_{k_{1}}$ and $\varphi A_{k_{1}}$ have a common circumellipse centered at 0 and intertwined vertices, also when $k_{1}=0$ (Figure 8).

As $\tau_{2} A_{k}=g_{k}^{2} A_{k}$, the offset and all harmonics are eigenvectors of $\tau_{2}, A_{k_{1}}$ is a fixed point,

$$
\lim _{m \rightarrow \infty} \tau_{2 m} P=A_{k_{1}}, \quad \text { and } \quad \lim _{m \rightarrow \infty} \tau_{2 m+1} P=\varphi A_{k_{1}} .
$$

In other words, the classical result (3) says that the offset or harmonic $A_{k_{1}}$ in the eigenspace of the dominant eigenvalue 1 of $\varphi^{*} \circ \varphi$ and $\varphi A_{k_{1}}$ can be asymptotically isolated by iterating the transformation. Figure 8 illustrates the case where the admissible filter $\varphi$ kills $F_{0}$ and has otherwise the spectral response of the normalized left midpoint filter $\sec \frac{\pi}{N} \cdot \sigma_{\ell} / 2$ given by (2).

Equation (3) is the key result. Theorem 1 follows now almost immediately from the fact that the above $A_{k_{1}}$, if nonzero, is a multiple of the sine table $\operatorname{Im} F_{k_{1}}$ when the polygon is a pseudo-sine! Indeed, due to symmetries, the spectrum of a pseudo-sine $S=\left(s_{j}\right)_{0 \leq j \leq N-1}$ of period $N=4 n$ is given, as is simply verified, by

$$
\begin{gathered}
\hat{s}_{2 k}=0, \quad \hat{s}_{2 k+1}=\frac{1}{i n} \sum_{j=1}^{n-1} s_{j} \sin \frac{j(2 k+1) \pi}{2 n}+\frac{1}{2 i n}(-1)^{k} s_{n}, \\
\hat{s}_{4 n-(2 k+1)}=-\hat{s}_{2 k+1}, \quad 0 \leq k \leq 2 n-1 .
\end{gathered}
$$

A pseudo-sine is thus the sum of its odd-numbered harmonics and

$$
A_{2 k+1}=2 i \hat{s}_{2 k+1} \operatorname{Im} F_{2 k+1}, \quad 0 \leq k \leq 2 n-1
$$

has collinear vertices and is proportional to the sine table

$$
T_{2 k+1}=\operatorname{Im} F_{2 k+1}=\left(\sin \frac{j(2 k+1) \pi}{2 n}\right)_{0 \leq j \leq 4 n-1} .
$$



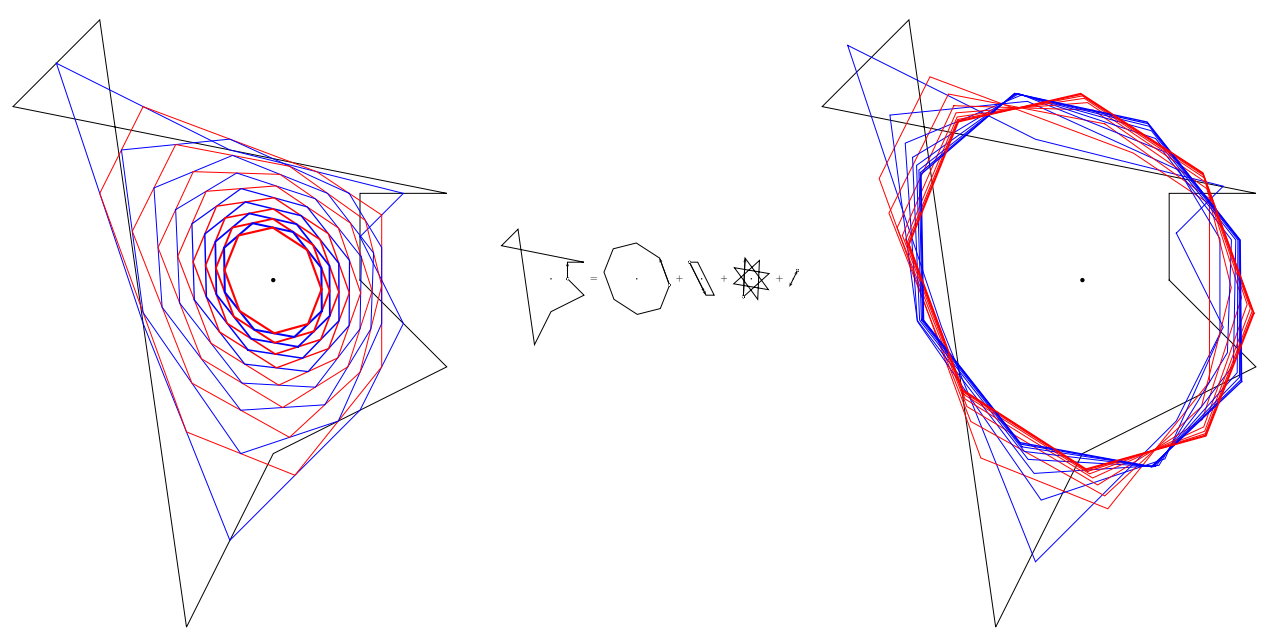

FIGURE 8. The Darboux filter: successive mean polygons of a central octagon and their normalizations

The fundamental is in particular proportional to the sought table $T_{1}=\left(\sin \frac{j \pi}{2 n}\right)_{0<j<4 n-1}$ and $T_{2 n-1}$ is $T_{1}$ with alternating signs. The fundamental does not vanish if and only if $\hat{s}_{1} \neq 0$ : this is for example ensured when the vertices $s_{1}$ to $s_{n}$ are real, nonnegative, and not all zero.

We can now formulate and prove our central result, Theorem 2, which is valid for any admissible filter and contains as special cases the Bürgi algorithm and its companions of Theorem 1.

Theorem 2. Let $\varphi$ be an admissible filter defined on $4 n$-gons and $S$ a pseudo-sine of period $N=4 n$ with nonzero $A_{k_{1}}$ (Definitions 1 and 2). The sine table $T_{k_{1}}=\operatorname{Im} F_{k_{1}}$ divided by any nonzero entry \# $j_{0}$ is the limit for $m \rightarrow \infty$ of any nonzero multiple of $\tau_{2 m} S$ divided by its vertex $\# j_{0}$. And the sine table $\operatorname{Im}\left(e^{i \eta_{k_{1}}} F_{k_{1}}\right)$ divided by any nonzero entry $\# j_{0}$ is the limit of any nonzero multiple of $\tau_{2 m+1} S$ divided by its vertex $\# j_{0}$.

Proof. As $A_{k_{1}}=2 i \hat{s}_{k_{1}} T_{k_{1}}$ by (5), here with $\hat{s}_{k_{1}} \neq 0$, one has by (3) $\lim _{m \rightarrow \infty} \tau_{2 m} S=$ $2 i \hat{s}_{k_{1}} T_{k_{1}}$, which can be divided by its nonzero value at vertex $\# j_{0}$ to give

$$
\lim _{m \rightarrow \infty} \frac{\tau_{2 m} S}{\text { its vertex } \# j_{0}}=\frac{T_{k_{1}}}{\text { its vertex } \# j_{0}} .
$$

As $\tau_{2 m+1} S=\varphi\left(\tau_{2 m} S\right)$ tends to $2 i \hat{s}_{k_{1}} \varphi T_{k_{1}}=\hat{s}_{k_{1}} \varphi\left(F_{k_{1}}-\overline{F_{k_{1}}}\right)=2 i \hat{s}_{k_{1}} \operatorname{Im}\left(e^{\eta_{k_{1}}} F_{k_{1}}\right)$, the same argument completes the proof.

In the Bürgi case, Theorem 1 follows at once from Theorem 2 by taking the admissible filter $\varphi=2 \sin \frac{\pi}{N} \cdot \int_{r}$, hence $k_{1}=1$ and $\eta_{1}=\frac{(2 n-1) \pi}{4 n}$, and noting that $\left(\int_{\ell} \circ \int_{r}\right)^{\circ m} S$ of the Bürgi steps is proportional to $\tau_{2 m} S$. In the Darboux-Nicollier case, the filter is $\varphi=\frac{1}{2} \sec \frac{\pi}{N} \cdot \sigma_{\ell}^{\prime}$, where the frequency response of $\sigma_{\ell}^{\prime}$ is given by 0 for $F_{0}$ and by the frequency response (2) of $\sigma_{\ell}$ otherwise, so that $\sigma_{\ell}^{\prime}$ and $\sigma_{\ell}$ are identical on central polygons as well as $\sigma_{\ell}^{\prime *}$ and $\sigma_{\ell}^{*}=\sigma_{r}$; here $k_{1}=1$ and $\eta_{1}=-\frac{\pi}{N}$ (Figure 8 shows an initial octagon that is not a pseudo-sine). In the inverse Bürgi case, we take $\varphi=\frac{1}{2} \csc \frac{(2 n-1) \pi}{4 n} \cdot \partial_{\ell}^{\prime}$, where the frequency response of $\partial_{\ell}^{\prime}$ is that of $\partial_{\ell}$ for the odd-numbered $F_{k}$ and 0 for the 
even-numbered ones, so that $\partial_{\ell}^{\prime}$ and $\partial_{\ell}$ are identical on pseudo-sines as well as $\partial_{\ell}^{\prime *}$ and $\partial_{\ell}^{*}=\partial_{r}$ on pseudo-cosines; here $k_{1}=2 n-1$ and $\eta_{1}=\frac{\pi}{N}$. In all three cases, $\varphi^{*} \circ \varphi$ is a bijective transformation of the subspace of pseudo-sines and is there uniquely determined by its restriction to the cores in the window $1 \leq j \leq n$ : these are the Bürgi, DarbouxNicollier, and inverse Bürgi steps, which describe the action of the corresponding filters on pseudo-sines only.

By Theorem 1, the $m$ th step gives twice as much approximate sine values as planned, for all integer multiples of the angle $\frac{\pi}{4 n}$ instead of $\frac{\pi}{2 n}$, if one considers both last lines. The needed approximation of $\cos \frac{\pi}{4 n}$ or $\sin \frac{\pi}{4 n}$, that is, of $\sqrt{1 \pm \cos \frac{\pi}{2 n}} / \sqrt{2}$, can be retrieved from the fact that, as limit for $m \rightarrow \infty$,

$$
\cos \frac{\pi}{2 n} \approx\left|\frac{\text { next to last entry }}{\text { last entry of the last line }}\right| .
$$

By Remark 1, the harmonics $A_{k_{1}}$ and $\varphi A_{k_{1}}$ are the images of $F_{k_{1}}$ and $e^{i \eta_{k_{1}}} F_{k_{1}}$ under the same affinity, for any polygon $P$. In the Bürgi case, the vertices of $A_{1}$ and $\varphi A_{1}$ on the common circumellipse coincide thus as sets if and only if $N \equiv 2(\bmod 4)$, and the $2 N$ vertices are images of the $(2 N)$ th roots of unity if and only if $N$ is a multiple of 4 . In the Darboux case, the vertices of $A_{1}$ and $\varphi A_{1}$ are images of the alternate $(2 N)$ th roots of unity (Figure 8).

\section{RATE OF CONVERGENCE}

Theorem 3 describes for our particular case the well-known rate of convergence of iterated linear diagonal operators with strictly dominating eigenvalue 1.

Theorem 3. If $\varphi$ is admissible (Definition 2), either $\left(\varphi^{*} \circ \varphi\right)^{\circ \mathrm{m}} P$ reaches $A_{k_{1}}$ in at most one step or the rate of convergence is the maximal gain $g_{k_{2}}^{2}$ of the nonzero harmonics of $P-A_{k_{1}}$ under $\varphi^{*} \circ \varphi$.

Proof. Either $\tau_{2} P=A_{k_{1}}$ or the norm of the nonzero error polygon $\tau_{2}^{\circ m} P-A_{k_{1}}$ is asymptotically (or exactly) multiplied by $g_{k_{2}}^{2}<1$ at each step $\tau_{2}=\varphi^{*} \circ \varphi$.

All even-numbered harmonics of a pseudo-sine $S=\left(s_{j}\right)_{0 \leq j \leq 4 n-1}$ of period $N=4 n$ vanish by (4). For the Darboux and inverse Bürgi filters, the rate of convergence to $A_{2 n-1}$ and $A_{1}$, respectively (see (3), (5), (6)), is poor in general since the difference between 1 and the next smaller gain is tiny (but it works nevertheless, see Figure 8!).

The situation is better for the Bürgi filter: if the first nonzero overtone of $S$ is $A_{k_{2}}$, the rate of convergence to $A_{1}=2 i \hat{s}_{1} T_{1}$ is

$$
g_{k_{2}}^{2}=\frac{\sin ^{2} \frac{\pi}{N}}{\sin ^{2} \frac{k_{2} \pi}{N}} \approx \frac{1}{k_{2}^{2}}
$$

which is at least about $\frac{1}{9}$. The convergence to $T_{1}$ is faster as soon as the first odd-numbered Fourier coefficients of $S$ behind $\hat{s}_{1} \neq 0$ are zero. Here are four such examples (a)-(d) with nonnegative integer or quadratic integer initial pseudo-sine cores $\left(s_{j}\right)_{1 \leq j \leq n}$, as is easily verified by direct computation of the Fourier coefficients (4).

(a) For $n=3 \nu$, the core $\Gamma$ with unique nonzero vertices $s_{\nu}=1$ and $s_{n}=2$ achieves $k_{2}=5$, as does the core $\Gamma^{\prime}$ with unique nonzero vertex $s_{2 \nu}^{\prime}=1$. The core $\Gamma+\sqrt{3} \Gamma^{\prime}$ reaches $k_{2}=11$ as soon as $\nu \geq 2$.

(b) The Bürgi step applied to the preceding core $\Gamma$ gives

$$
(2,4,6, \ldots, 2 \nu, 2 \nu+1,2 \nu+2, \ldots, 4 \nu) \text { for } n=3 \nu .
$$


For $n=9$, this is Bürgi's original core (Table 1), which therefore also achieves $k_{2}=5$ [30].

(c) For $n=15 \nu$, the core with unique nonzero vertices $s_{2 \nu}=s_{10 \nu}=s_{12 \nu}=1$ achieves $k_{2}=7$.

(d) For $n=15 \nu$, the core $\Gamma$ with unique nonzero vertices $s_{\nu}=s_{11 \nu}=1$ achieves $k_{2}=7$ and $\hat{s}_{9}=0$, as does the core $\Gamma^{\prime}$ with unique nonzero vertices $s_{7 \nu}^{\prime}=s_{13 \nu}^{\prime}=1$. As

$$
\frac{\sin 42^{\circ}+\sin 78^{\circ}}{\sin 6^{\circ}+\sin 66^{\circ}} \text { is the golden ratio } \rho=\frac{1+\sqrt{5}}{2}=2 \cos 36^{\circ},
$$

the core $\Gamma+\rho \Gamma^{\prime}$ reaches $k_{2}=11$. If $f_{\mu}$ and $f_{\mu+1}$ are two fixed consecutive Fibonacci numbers, the initial core $f_{\mu} \Gamma+f_{\mu+1} \Gamma^{\prime}$ reaches only $k_{2}=7$, but the norm of the error polygon is divided by about 121 at each of the first steps as $f_{\mu+1} / f_{\mu}$ approximates the golden ratio.

Consider in particular $n=90$, as in Bürgi's sine table for all degrees, and the core $\Gamma$ with unique nonzero vertices $s_{6}=s_{66}=1$. Five Bürgi steps with integer vertices and a division of the final core by its last vertex, which is about $8.6 \times 10^{15}\left(<60^{9}\right)$, provide approximate sine values for all degrees with a maximal deviation of $1.1 \times 10^{-8}$. The next to last half Bürgi step gives the sines of the half degrees within $7.7 \times 10^{-8}$ by using the aforementioned estimation (7) of $\cos 0.5^{\circ}$, which is too large by $10^{-11}$.

It is even possible to avoid final divisions by the last core vertex! For $n=90$, the initial core $\widetilde{\Gamma}$ with unique nonzero vertices

$$
\widetilde{s}_{1}=1526986833984733 \text { and } \widetilde{s}_{90}=21502961542631
$$

leads after five Bürgi steps to a core $\left(\widetilde{s}_{5, j}\right)_{1 \leq j \leq 90}$ with last vertex $2^{98}$ : the values $\sin \ell^{\circ}$, $1 \leq \ell \leq 90$, are approximated within $5 \times 10^{-5}$ by this final core divided by $2^{98}$ and the computation in binary arithmetic requires only integer additions and bit shifts. How did we choose $\widetilde{\Gamma}$ ? When the only nonzero core vertices are $\widetilde{s}_{1}$ and $\widetilde{s}_{90}$, one finds for the last vertex $\widetilde{s}_{5,90}$

$$
4 \widetilde{s}_{5,90}=591510389403645 \widetilde{s}_{1}+16947527098590161 \widetilde{s}_{90}
$$

with coprime coefficients. The extended Euclidean algorithm leads then to the above positive integer solutions $\widetilde{s}_{1}$ and $\widetilde{s}_{90}$ of $4 \widetilde{s}_{5,90}=2^{100}$.

In the same way, the core with unique nonzero vertices

$$
s_{1}=32529867920854824 \text { and } s_{90}=1224853929315320
$$

leads to $s_{5,90}=10^{31}$ (using only integers) and the division of the final core by $10^{31}$ provides approximations of $\sin \ell^{\circ}, 1 \leq \ell \leq 90$, within $2.4 \times 10^{-5}$.

\section{NAPOLEON'S THEOREM REVISITED}

To further illustrate the efficiency of the spectral approach, we give a very short and completely formula-free proof of two celebrated theorems: Napoleon's theorem for triangles $[14,26]$ and its generalization, the Petr-Douglas-Neumann theorem for planar polygons $[23,6,15,16,3,26]$. They provide in fact a geometric way of retrieving the spectral decomposition of a polygon. Napoleon has nothing to do with his theorem and Jesse Douglas (1897-1965) was one of the two first Fields medalists in 1936. We already presented similar simple proofs in $[17,20]$ and found no other comparable references in the literature.

According to the Fourier decomposition, every triangle is the sum $E_{+}+E_{-}$of a positively and a negatively oriented equilateral triangles (possibly trivial). If one erects on the 


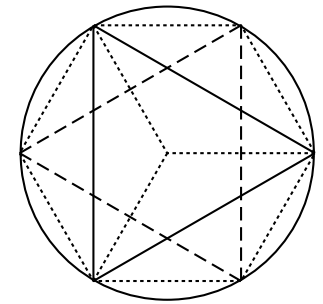

(A)

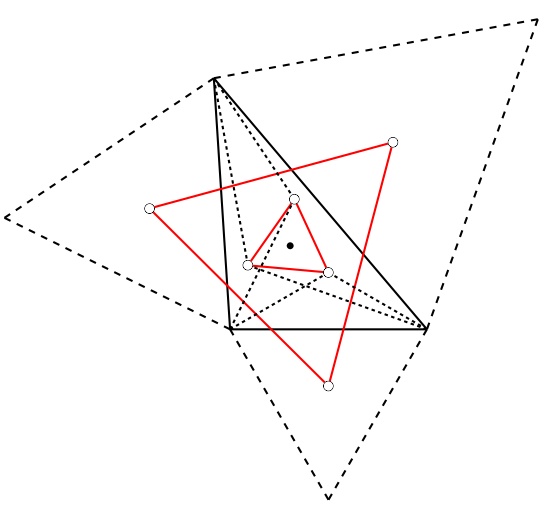

(B)

FIGURE 9. Napoleon's theorem

successive sides of a triangle right-hand isosceles ears with apex angle $\frac{2 \pi}{3}$ and takes the triangle of their apices, $E_{+}$is simply rotated by $\frac{\pi}{3}$ about its center and $E_{-}$killed (Figure 9a). The transformation with corresponding left-hand ears kills $E_{+}$and rotates $E_{-}$ by $-\frac{\pi}{3}$. Admit for the moment that these filters are linear (proof below) and Napoleon's theorem is established (Figure $9 b$ )!

Theorem 4 (Napoleon's). The centers of three right-hand and three left-hand equilateral ears erected on the successive sides of any triangle are the vertices of a positively and a negatively oriented equilateral triangle (possibly trivial), respectively.

Any central triangle is thus the sum $\hat{z}_{1} F_{1}+\hat{z}_{2} F_{2}$ of its positively and negatively oriented Napoleon triangles rotated by $-\frac{\pi}{3}$ and $\frac{\pi}{3}$ about the origin.

Here is the generalization to $N$-gons. For $1 \leq q \leq N-1$, define the filter $\varepsilon_{q}$ as follows: consider the triangle formed by an oriented side of $F_{q}$ and the origin ( $F_{q}$ 's center) as third vertex, erect ears directly similar to this triangle on the successive sides of any input $N$-gon and take as output the polygon of the successive ear apices. Due to regularity of the Fourier basis polygons, $\varepsilon_{q}$ multiplies $F_{k}$ by the first side's ear apex, which is zero only for $F_{q}$. The filter $\varepsilon_{q}$ is linear as each ear apex is the image of the side endpoint under a spiral similarity centered at the side's starting point with constant dilation and rotation. This proves the final Theorem 5.

Theorem 5 (Petr-Douglas-Neumann). For any polygon $F_{k} \neq F_{0}$ of the Fourier basis, let $\varepsilon_{k}$ be the filter whose output's vertices are the centers of the scaled and rotated copies of $F_{k}$ erected on the successive sides of the input $N$-gon. Successive filtering by all $\varepsilon_{k}$ but $\varepsilon_{q}$ transforms any $N$-gon into an output directly similar to $F_{q}$ or trivial; the result is independent of the filtering order.

The frequency response of $\varepsilon_{q}$ has a nice geometric interpretation. The ears of $\varepsilon_{q}$ are directly similar to the normalized ear $\left(0,1, a_{q}\right)$ with $a_{q}=\frac{1}{2}\left(1+i \cot \frac{q \pi}{N}\right)$. The frequency response of $\varepsilon_{q}$, that is, the vertex of the ear erected on the first side $1 \rightarrow e^{i k \frac{2 \pi}{N}}$ of $F_{k}$, is thus

$$
H_{q, k}=1+a_{q}\left(e^{i k \frac{2 \pi}{N}}-1\right)=\overline{a_{q}}+a_{q} e^{i k \frac{2 \pi}{N}}, \quad 0 \leq k \leq N-1,1 \leq q \leq N-1 .
$$

Hence the frequency response polygon $\left(H_{q, k}\right)_{0 \leq k \leq N-1}$ of $\varepsilon_{q}$ is the regular $N$-gon directly similar to $F_{1}$ with vertex $\# 0$ at 1 and vertex $\# q$ at 0 (and center $\overline{a_{q}}=a_{N-q}$ ): for $N=4$, 
these are the squares $(1,0,-i, 1-i)$ for $\varepsilon_{1},\left(1, \frac{1+i}{2}, 0, \frac{1-i}{2}\right)$ for $\varepsilon_{2}$, and $(1,1+i, i, 0)$ for $\varepsilon_{3}$. If $N$ is even, $\varepsilon_{N / 2}$ is the midpoint filter $\sigma_{r} / 2$ we already met (Figure 8).

As the product of all factors $H_{k, q}, k \neq q$, is $e^{i\left(\pi-q \frac{2 \pi}{N}\right)}$ [17], the opposite of vertex \#1 in the output of Theorem 5 coincides, for a central input $P=\left(z_{j}\right)_{0 \leq j \leq N-1}$, with the initial vertex $\hat{z}_{q}$ of $\hat{z}_{q} F_{q}$, the orthogonal projection of $P$ on $F_{q}$ (Figure 7): $\hat{z}_{q} F_{q}$ can now be retrieved.

\section{CONCLUSiON}

Bürgi's achievement really deserves its name Artificium, the skillful method! And the Fourier decomposition provides a proof that is up to it and establishes its close relation to Napoleon's theorem: an asymptotic extraction of a harmonic versus an exact extraction of Fourier components. If one transforms an initial polygon alternately with an admissible filter and its adjoint, the alternate outputs converge to the harmonic (or offset) corresponding to the maximal gain 1 and to its image under the filter, both limits having the same circumellipse. When the initial polygon is a pseudo-sine, these limits, if nonzero, are proportional to sine tables. And the sine tables can be retrieved by normalizing the limits appropriately. The study of the iterated inverse Darboux-Nicollier step is left to the reader!

\section{REFERENCES}

[1] E. R. Berlekamp, E. N. Gilbert, and F. W. Sinden: A Polygon Problem, Am. Math. Mon. 72 (1965) 233-241.

[2] J. Bürgi: Fundamentum Astronomia, 1592. Digital Library of the University of Wrocław. http://www.bibliotekacyfrowa.pl/dlibra/doccontent?id=45624

[3] G. Chang and P. J. Davis: A Circulant Formulation of the Napoleon-Douglas-Neumann Theorem, Linear Algebra Appl. 54 (1983) 87-95.

[4] K. Clark: Jost Bürgi's Aritmetische und geometrische Progreß Tabulen (1620). Edition and commentary. Science Networks, Historical Studies 53. Birkhäuser/Springer, New York, NY, 2015.

[5] G. Darboux: Sur un problème de géométrie élémentaire, Bull. Sci. Math. Astr. $2^{e}$ Sér. 2 (1878) 298-304. http://archive.numdam. org/ARCHIVE/BSMA/BSMA_1878_2_2_1/BSMA_1878_2_2_1_298_1/BSMA_1878_2_2_1_298_1.pdf

[6] J. Douglas: On Linear Polygon Transformations, Bull. Am. Math. Soc. 46 (1940) 551-560.

[7] J. C. Fisher, D. Ruoff, and J. Shilleto: Perpendicular Polygons, Am. Math. Mon. 92 (1985) 23-37.

[8] M. Folkerts: Eine bisher unbekannte Schrift von Jost Bürgi zur Trigonometrie, 107-114. In: R. Gebhardt (ed.), Arithmetik, Geometrie und Algebra in der frühen Neuzeit. Adam-Ries-Bund, Annaberg-Buchholz, 2014.

[9] M. Folkerts, D. Launert, and A. Thom: Jost Bürgi's Method for Calculating Sines, Hist. Math. 43(2) (2016) 133-147.

[10] B. Grünbaum: Lecture Notes on Modern Elementary Geometry. EPrint Collection, University of Washington, 1999. http://hdl. handle.net/1773/15592

[11] J. Kepler: Gesammelte Werke. Bayerische Akademie der Wissenschaften, Munich, 1937-2017. http://kepler.badw.de/kepler-digital.html

[12] D. Launert: Nova Kepleriana: Bürgis Kunstweg im Fundamentum Astronomiæ, Entschlüsselung seines Rätsels. Verlag der Bayerischen Akademie der Wissenschaften, Munich, 2015.

[13] M. List and V. Bialas: Nova Kepleriana: Die Coss von Jost Bürgi in der Redaktion von Johannes Kepler. Verlag der Bayerischen Akademie der Wissenschaften, Munich, 1973.

[14] H. Martini: On the Theorem of Napoleon and Related Topics, Math. Semesterber. 43 (1996) 47-64.

[15] B. H. Neumann: Some Remarks on Polygons, J. London Math. Soc. 16 (1941) 230-245.

[16] B. H. Neumann: A Remark on Polygons, J. London Math. Soc. 17 (1942) 165-166.

[17] G. Nicollier: Convolution Filters for Polygons and the Petr-Douglas-Neumann Theorem, Beitr. Algebra Geom. 54 (2013) 701-708.

[18] G. Nicollier: Convolution Filters for Triangles, Forum Geom. 13 (2013) 61-85.

[19] G. Nicollier and A. Stadler: Limit Shape of Iterated Kiepert Triangles, Elem. Math. 68 (2014) 61-64.

[20] G. Nicollier: Some Theorems on Polygons With One-line Spectral Proofs, Forum Geom. 15 (2015) 267273.

[21] G. Nicollier: A Characterization of Affinely Regular Polygons, Beitr. Algebra Geom. 57 (2016) 453-458. 
[22] P. Pech: The Harmonic Analysis of Polygons and Napoleon's Theorem, J. Geom. Graph. 5 (2001) 13-22.

[23] K. Petr: O jedné větě pro mnohoúhelníky rovinné (On a Theorem for Planar Polygons), Čas. Mat. Fys. 34 (1905) 166-172 . http://www.digizeitschriften.de/dms/toc/?PPN=PPN31311028X_0034

[24] D. Roegel: Some remarks on Bürgi's interpolations. Technical report, LORIA, Nancy, 2016. http://locomat.loria.fr/locomat/reconstructed.html

[25] I. J. Schoenberg: The Finite Fourier Series and Elementary Geometry, Am. Math. Mon. 57 (1950) 390-404.

[26] W. Schuster: Polygonfolgen und Napoleonsätze, Math. Semesterber. 41 (1994) 23-42.

[27] D. B. Shapiro: A Periodicity Problem in Plane Geometry, Am. Math. Mon. 91 (1984) 97-108.

[28] F. Staudacher: Jost Bürgi, Kepler und der Kaiser. Revised and enlarged 3rd ed. NZZ Libro, Zurich, 2016.

[29] D. Vartziotis and J. Wipper: On the Construction of Regular Polygons and Generalized Napoleon Vertices, Forum Geom. 9 (2009) 213-223.

[30] J. Waldvogel: Jost Bürgi's Artificium of 1586 in Modern View, an Ingenious Algorithm for Calculating Tables of the Sine Function, Elem. Math. 71 (2016) 89-99.

UNIVERSITY OF APPLIED SCIENCES OF WESTERN SWITZERLAND

ROUTE DU RAWIL 47, CH-1950 SION, SWITZERLAND

E-mail address: gregoire.nicollier@hevs.ch 